\title{
Quantitative assessment of metamorphopsia using M-charts and Amsler tests in patients with non-treated central serous chorioretinopathy
}

\author{
Edyta Koman, Dorota Borowicz, Małgorzata Ozimek, Agnieszka Oleszczuk, \\ Katarzyna Nowomiejska, Robert Rejdak \\ Department of General Ophthalmology Medical University in Lublin, Lublin, Poland
}

\begin{abstract}
INTRODUCTION. The aim of this study is the assessment of the clinical parameters related to metamorphopsia using M-charts and traditional Amsler tests in patients with central serous chorioretinopathy (CSCR).

MATERIALS AND METHODS. Seventeen eyes of 17 patients were examined with M-charts and Amsler tests, as well as with ocular coherence tomography (OCT) at each of three visits during four months of follow-up. The rate of metamorphopsia detection was $82 \%$ with M-charts and $76 \%$ with Amsler test.

RESULTS. The vertical metamorphopsia score decreased after four months in nine patients (52.9\%), $0.1^{\circ}$ on average; increased in two patients $(11.8 \%)$, mean $0.2^{\circ}$; and there was no change in six patients (35.3\%). The horizontal metamorphopsia score decreased in seven patients $(41.2 \%), 0.2^{\circ}$ on average, increased in three patients $(17.6 \%)$, $0.23^{\circ}$ on average, and there was no change in seven patients $(41.2 \%)$.

CONCLUSIONS. M-charts are superior to the traditional Amsler charts in the monitoring of metamorphopsia in patients with CSCR. M-charts are a good diagnostic tool for quantitative assessment of metamorphopsia and monitoring over time in patients with CSCR.
\end{abstract}

KEY WORDS: metamorphopsia, M-charts, central serous chorioretinopathy

Ophthalmol J 2016; Vol. 1, No. 3, 89-92

\section{INTRODUCTION}

Central serous chorioretinopathy (CSCR) is characterised by localised and limited serous detachments of the neurosensory retina associated with leakage at the level of the retinal pigment epithelium (RPE). The term "central" refers to the form of the disease causing visual symptoms due to the presence of serous detachments in the macular area [1]. The symptoms of the visual loss in this disease include metamorphopsia, micropsia, dyschromatopsia, scotoma, and reduced contrast sensitivity [2]. In most of the patients CSCR is a benign and self-limiting disease with spontaneous resolution of serous retinal detachment (SRD) and recovery of good vision. However, in some patients visual acuity can be worse even after retinal reattachment [1]. Asymptomatic subjects may have presented one or multiple episodes of extra macular serous detachments, as often observed in the contralateral eye of an active CSCR patient [3].

The exact molecular mechanisms of CSCR have remained uncertain. Several risk factors for the development of CSCR exist, but only high serum glucocorticoid and serum catecholamine levels have been related to the occurrence of CSCR $[4,5]$. Further risk factors that have been proposed include 
pregnancy, alcohol consumption, untreated hypertension, use of antibiotics, bone marrow or organ transplantation, infection of the respiratory tract, and infection with Helicobacter pylori $[4,6]$.

Metamorphopsia is a major sign in patients with macular diseases and is defined as a deformation of seen straight lines due to the displacement of photoreceptors. Metamorphopsia is a major visual symptom in patients with CSCR and often leads to poor visual quality, even after recovery to a good visual acuity. Several tools have been developed for quantification of metamorphopsia, including preferential hyperacuity perimetry (PHP) and the Amsler test [7]. The M-chart is a diagnostic tool developed by Matsumoto [8] to quantify the degree of metamorphopsia in patients with macular diseases.

The aim of this study was to determine the clinical parameters related to metamorphopsia in patients with non-treated CSCR.

\section{METHODS}

This prospective study was conducted in the medical retina out-patents clinic of the Department of General Ophthalmology of the Medical University in Lublin, Poland. Informed consent was obtained from all of the subjects. We examined 17 eyes of 17 patients ( 15 men and 2 women) diagnosed with CSCR during three separate visits: after episode of CSCR (1st visit), after one month (2nd visit) and three months (3rd visit). Each patient had a documented episode of CSCR confirmed by fluorescein angiography (FA) (HRA, Heidelberg Engineering, Germany) and optical coherence tomography (OCT) (Cirrus, Zeiss, US). We excluded patients with the following retinal diseases: age-related macular degeneration, idiopathic choroidal neovascularisation (CNV), epiretinal membrane (ERM), vitreoretinal traction, and intraocular inflammation. Additionally, we excluded patients taking any systemic corticosteroids.

Each patient had full ophthalmic examination including measurement of the best-corrected visual acuity (BCVA), slit-lamp biomicroscopy, fundus examination after dilatation, Amsler test, M-charts, and OCT including central retinal thickness (CRT).

The M-charts (Inami Co., Tokyo, Japan), consist of 19 dotted (dot size is $0.1^{\circ}$ ) lines with dot intervals ranging from $0.2^{\circ}$ to $2.0^{\circ}$ of visual angle [9]. The examination is performed at a distance of $30 \mathrm{~cm}$ with appropriate near correction. At first, a vertical straight line $\left(0^{\circ}\right)$ on the first page of M-charts is shown to the patient. If the patients recognise the straight line as straight, the metamorphopsia score is 0 . If a patient recognises the straight line as an irregular or curved line, more coarse dotted lines are shown to the patient in the following test. When the patient recognises a dotted line as straight, its visual angle is considered as his/her metamorphopsia score - a minimum visual angle of a dotted line needed to detect metamorphopsia [10]. Next, the M-charts are rotated by $90^{\circ}$ and the same test is performed using horizontal lines. In this study the M-chart test was performed both with horizontal $(\mathrm{MH})$ and vertical (MV) lines to obtain separate $\mathrm{MH}$ and $\mathrm{MV}$ $\mathrm{M}$-charts scores. The M-chart result was considered positive if the metamorphopsia score was more than $0^{\circ}\left(0.2-2.0^{\circ}\right)$. If it was $0^{\circ}$, it was considered as negative. This assessment was done in regard to $\mathrm{VH}$ and $\mathrm{MH}$ separately and for both methods together - it was considered as positive if one of them was positive, and negative if both were negative $[9,11]$. The Amsler test was positive if any blurred, wavy, or broken lines were reported by the patient. The OCT result was positive if a subretinal fluid was detected.

\section{RESULTS}

There were 15 men (88.2\%) and 2 women $(11.8 \%)$ in the study group. The mean age was 40.4 years (range $24-53$ years). The mean age of men was 41 and of women was 36 years. The rate of detection of the metamorphopsia (percentage of positive results) of MV was $76.5 \%$ on the first visit and $52.9 \%$ on the third visit, $\mathrm{MH}-70.6 \%$ and $47.1 \%$, respectively, both together $-73.6 \%$ and $50 \%$; the sensitivity of M-charts was $82 \%$. The MV score decreased after four months in nine patients $(52,9 \%), 0.1^{\circ}$ on average; it increased in two patients $(11.8 \%)$, mean $0.2^{\circ}$; there was no change in six patients $(35.3 \%)$. The $\mathrm{MH}$ score decreased in seven patients $(41.2 \%), 0.2^{\circ}$ on average, increased in three patients $(17.6 \%), 0.23^{\circ}$ on average, and there was no change in seven patients $(41.2 \%)$. The median of the MV score was $0.38^{\circ}$ (range $0-2.0^{\circ}$ ). The median of the $\mathrm{MH}$ score was $0.34^{\circ}$ (range $0-2.0^{\circ}$ ). The Amsler grid results improved in six patients, deteriorated in two patients, and remained the same in nine patients. The sensitivity of Amsler test was $76 \%$. The mean BCVA improved from 0.65 on the first visit to 0.78 on the third visit. The visual acuity in the near Snellen test improved from 1.0 to 0.71 . The median CRT in OCT was $380.1 \mu \mathrm{m}$ on the first visit and $290.2 \mu \mathrm{m}$ on the third visit. CRT improved in 13 patients. 


\section{DISCUSSION}

CSCR is a major cause of vision threat among middle-aged men. A large number of single studies suggest that men are more often affected than women, presenting male-to-female ratios of up to 8:1 [4]. The mean age of patients with the acute form of this disease has a peak at around $40-45$ years but in patients with chronic CSCR the mean age is higher $[4,13]$. The clinical diagnosis in older patients is more difficult because chronic CSCR often resembles age-related macular degeneration (AMD) or can be complicated by choroidal neovascularisation (CNV) $[1,13,14]$.

Metamorphopsia is a significant symptom of CSCR. M-charts may be used as a diagnostic tool in the detection of metamorphopsia in CSCR patients, it is possible to monitor quantitatively the changes in the degree of metamorphopsia over time. Matsumoto et al. [8] reported that the dotted lines of M-charts are designed to evaluate the fixation area because metamorphopsia can be noticed in the fixation area. The median of the MV score decreased from $0.45^{\circ}$ to $0.22^{\circ}$ from first to third visit and the median of the $\mathrm{MH}$ score decreased from $0.44^{\circ}$ to $0.31^{\circ}$.

The Amsler grid, consisting of evenly spaced horizontal and vertical lines, has been widely used since 1947 to test metamorphopsia [15]. The Amsler grid can help detect one of the first signs of retinal disease as wavy, broken, or distorted lines or a blurred or missing area of vision. It is very cheap and easily understood by the patient. However, it also produces high false negative rates and does not allow for the quantification of the severity of metamorphopsia [9]. OCT is an accurate tool for qualitative and quantitative assessment of the macular structure. This device might show the precise structural changes of the macular region, like pigment epithelial detachment (PED), retinal pigment epithelium (RPE) irregularities, subretinal fluid, and CRT - distance between the anterior (internal limiting membrane) and posterior (retinal pigment epithelium) highly reflected boundaries of the retina [10]. The M-charts are superior to the Amsler grid in detecting metamorphopsia in patients with retinal disease, for example CSCR, AMD, or ERM. Amsler's charts detect distortion in place, while M-charts uses lines to assess distortion. Although Amsler's charts do not quantify the degree of distortion, the locations and shapes of distortion can be detected. M-charts can quantify the degree of distortion but cannot describe the shapes and locations of the subject's metamorphopsia [10]. The M-charts provide more information than the Amsler charts in regard to the horizontal and vertical lines. In our study the rate of metamorphopsia detection in patients with CSCR was higher with the M-charts (82\%) than with Amsler test (76\%). Possible explanations for this high false-negative rate are the filling phenomenon across pathological scotomas and the use a preferred retinal locus away from the scotoma boundary [16]. Therefore, the Amsler grid alone does not seem to be sufficient for metamorphopsia detection and for monitoring the changes over time [9].

The rate of metamorphopsia was quantified using M-charts, and clinical parameters including retinal (foveal) microstructures were monitored by the OCT images. Lehmann et al. [17] presented cohort study measurements, and $395 \mathrm{~mm}$ could be used as a sensitive value to diagnose "thick choroid" or "pachychoroid". Increased choroidal thickness is associated with CSCR, but it is not mandatory for its diagnosis. There is a reduction in choroidal thickness with age, axial length, circadian rhythm, and myopic refractive error, and the choroid is also thinner in females than in males [18]. Gotkas proved that affected eyes of unilateral cases were shown to have a greater choroidal thickness than fellow eyes [19].

In our research CRT on average decreased from $380 \mu \mathrm{m}$ to $290 \mu \mathrm{m}$, which testifies about regression subretinal fluid.

Fujita et al. [20] suggested that in CSCR, metamorphopsia results from disruption to the regular alignment of the photoreceptor layers followed by development of serous fluid. Subretinal fluid is associated with RPE leakage; other changes observed with OCT imaging have given insight into the pathogenesis of CSCR.

\section{CONCLUSIONS}

This study showed that the rate of metamorphopsia detection in CSCR patients was better with M-charts than with Amsler grid. There is a fast and easy method which detect metamorphopsia when Amsler chart is normal. M-charts give additional information about visual function, independent of the visual acuity and morphological changes in OCT. It can be used in the quantitative assessment of the progression of metamorphopsia in patients suffering from CSCR. 


\section{REFERENCES}

1. 1. Daruich A, Matet A, Dirani A et al. Central serous chorioretinopathy: Recent findings and new physiopathology hypothesis. PRER 2015; 48: 82-118.

2. Bae $\mathrm{S}$, Jin K, Kim H, Bae SH. Clinical parameters related to metamorphopsia outcome in patients with resolved central serous chorioretinopathy using M-CHARTS: retrospective cohort study. BMC Ophthalmology 2015; 15: 180.

3. Weenink AC, Borsje RA, Oosterhuis JA. Familial chronic central serous chorioretinopathy. Ophthalmologica 2001; 215: 183-187.

4. Liegl R, Ulbig MW. Central serosus chorioretinopathy. Ophthalmologica 2014; 232: 65-76.

5. Bouzas EA, Karadimas P, Pournaras CJ. Central serous chorioretinopathy and glucocorticoids. Surv Ophthalmol 2002; 47: 431-448.

6. Tittl MK, Spaide RF, Wong D et al. Systemic findings associated with central serous chorioretinopathy. Am J Ophthalmol 1999; 128: 63-68.

7. Arimura $\mathrm{E}$, Matsumoto $\mathrm{C}$, Nomoto $\mathrm{H}$ et al. Correlations between M-CHARTS and PHP findings and subjective perception of metamorphopsia in patients with macular diseases. Invest Ophthalmol Vis Sci 2011; 52: 128-135.

8. Matsumoto C, Arimura E, Hashimoto S, Takada S, Okuyama S, Shimomura Y. A new method for quantification of metamorphopsia using M-CHARTS (in Japanese). Rinsho Ganka 2000; 54: 373-377.

9. Nowomiejska K, Oleszczuk A, Brzozowska A et al. M-charts as a tool for quantifying metamorphopsia in age-related macular degeneration treated with the bevacizumab injections. BMC Ophthalmology 2013; 13: 13.

10. Nomoto H, Matsumoto C, Arimura E et al. Quantification of changes in metamorphopsia and retinal contraction in eyes with spontaneous separation of idiopathic epiretinal membrane. Eye 2013; 27: 924-930.
11. Bae SW, Chae JB. Assessment of metamorphopsia in patients with central serous chorioretinopathy. Indian J Ophthalmol 2013; 61: 172-175.

12. Kitzmann AS, Pulido JS, Diehl NN, Hodge DO, Burke JP. The incidence of central serous chorioretinopathy in Olmsted County, Minnesota, 1980-2002. Ophthalmology 2008; 115: 169-173.

13. Fung AT, Yannuzzi LA, Freund KB. Type 1 (sub-retinal pigment epithelial) neovascularization in central serous chorioretinopathy masquerading as neovascular age-related macular degeneration. Retina 2012; 32: 1829-1837.

14. Inhoffen W, Ziemssen F, Bartz-Schmidt KU. Chronic central serous chorioretinopathy (cCSC): differential diagnosis to choroidal neovascularisation (CNV) secondary to age-related macular degeneration (AMD). Klin 2012; 229: 889-896.

15. Amsler M. L'examen qualitative de la foction maculaire. Ophthalmologica 1947; 114: 248-261.

16. Crossland MD, Culham LE, Kabanarou SA. Preferred retinal locus development in patients with macular disease. Ophthalmology 2005; 112: 1579-1585.

17. Lehmann M, Bousquet $E$, Beydoun T, Behar-Cohen F. Pachychoroid: An inherited condition? Retina 2015; 35: 10-16.

18. Fujiwara T, Imamura Y, Margolis R, Slakter JS, Spaide RF. Enhanced depth imaging optical coherence tomography of the choroid in highly myopic eyes. Am J Ophthalmol 2009; 148: 445-450.

19. Goktas A. Correlation of subretinal fluid volume with choroidal thickness and macular volume in acute central serous chorioretinopathy. Eye 2014; 28: 1431-1436.

20. Fujita K, Imamura Y, Shinoda K et al. Quantification of metamorphopsia in chronic central serous chorioretinopathy after half-dose verteporfin photodynamic therapy. Retina 2014; 34: 964-970. 